\title{
"Painful legs and moving toes" associated with polyneuropathy
}

\author{
PASQUALE MONTAGNA, FABIO CIRIGNOTTA, TOMMASO SACQUEGNA, PAOLO \\ MARTINELLI, GIOVANNI AMBROSETTO, ELIO LUGARESI
}

From the Institute of Neurology, University of Bologna, Bologna, Italy

SUMMARY Three patients showing clinical and electrophysiological evidence of peripheral neuropathy also suffered from the syndrome of "painful legs and moving toes". Polygraphic recordings during wakefulness and sleep were consistent with the hypothesis that pathological afferent discharges arising peripherally may induce, via the spinal cord, motor excitation and pain. A severe disruption of the pattern of sleep was found in all cases.

A syndrome of "painful legs and moving toes", characterised by spontaneous causalgic pain in the lower limbs and associated with peculiar involuntary movements of the toes and foot, was first described by Spillane et al in $1971 .^{1}$ The syndrome has been observed after a variety of lesions affecting the posterior nerve roots, the spinal ganglia or the peripheral nerves. ${ }^{2-4}$ Reported here are the first polygraphic findings in "painful legs and moving toes", as observed in three patients affected with peripheral neuropathy who showed signs of this rare syndrome, with emphasis on the associated disruption of the pattern of sleep.

\section{Case reports}

Case 1 A 60-year-old former clerk had a history of peptic ulcer and, from age 32 years, of lung tuberculosis, treated with long-term streptomycin and isoniazid, complicated by knee arthropathy and right nephrectomy at the age of 39 years. At age 57 years, burning and heating pain began in both feet, continuous throughout the day, and causing severe insomnia. After an 8 month remission, the deep burning pain spread to involve the legs up to the knee and peculiar continuous, involuntary and irregular movements of all toes of both feet appeared at rest; they could be temporarily stopped by voluntary effort, but this increased the burning sensation. These symptoms progressively worsened during the next year, with painful electric-like sensations arising in the feet and back of legs. On examination, there was bilateral weakness of the extensor muscles of the ankles and toes, associated with muscular wasting in

Address for reprint requests: Dr P Montagna, Clinica Neurologica, Via Foscolo 7-40123 Bologna (Italy).

Received 21 July 1982 and in revised form 3 November 1982 Accepted 20 November 1982 the thighs, calves and feet, especially on the right, and with slight distal hypoesthesia. Both ankle jerks were absent. Dystrophic skin changes included redness of toes and toenails and a dry and scaly skin on both legs. There were rapid and irregular movements of all toes at rest, each toe moving independently of the others in flexion, extension or abduction. Radiography of the cervical and lumbar spine, blood nitrogen and glucose, full blood count, $\mathrm{Hb}$, urinalysis, liver function tests and WR were normal. Electromyography (EMG) showed signs of chronic partial denervation in the right tibialis anterior and flexor hallucis brevis muscles. Motor conduction velocity was decreased in the right peroneal nerve $(39 \mathrm{~m} / \mathrm{s})$; the sensory action potentials (SAPs) in the right superficial peroneal and right median nerves showed a markedly reduced amplitude $(0 \cdot 1$ $\mu \mathrm{V}$ and $0.6 \mu \mathrm{V}$ respectively); sensory conduction velocity was normal $(53 \mathrm{~m} / \mathrm{s}$ and $49 \mathrm{~m} / \mathrm{s}$ respectively). At rest, there were spontaneous discharges of motor unit action potentials, especially in the extensor digitorum brevis and abductor digit minimi muscles. These occurred in bursts at a frequency of about $0.5 \mathrm{~Hz}$, sometimes alternating in the two muscles (fig 1). One-hundred $\mathrm{Hz}$ vibration of the flexor hallucis muscle markedly increased the spontaneous discharge until it reached a reduced recruitment pattern, 2 $\mathrm{mV}$ amplitude. Acute iv administration of baclofen $10 \mathrm{mg}$ led, within 5 minutes, to the disappearance of the spontaneous EMG discharges and alleviated the pain, but chronic oral therapy with baclofen up to $100 \mathrm{mg}$ daily was ineffective. Carbamazepine, amitriptyline and benzodiazepines also proved to be unhelpful. The patient was very incapacitated by the pain and, to a lesser degree, by the distressful toe movements. He felt depressed and admitted to suicidal thoughts. A polygraphic study during the night on two separate occasions showed, during wakefulness, pseudo-rhythmic EMG bursts at rest, especially in the right abductor digiti minimi, extensor digitorum brevis, and abductor hallucis (fig 2). Toe movements were considerably reduced during slow-wave sleep (SWS), only a few isolated jerks sporadically arising in the foot or leg muscles. In contrast, a brief episode of REM sleep was charac- 


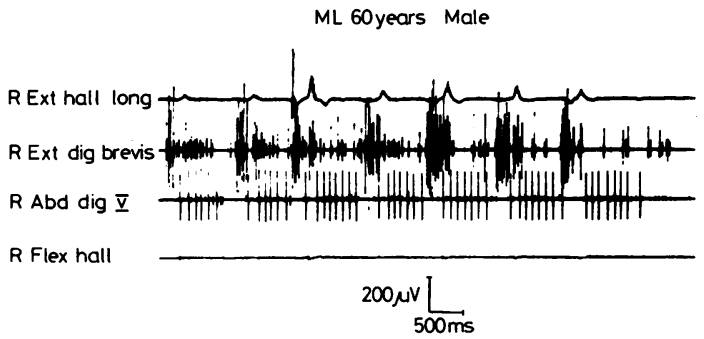

Fig 1 Case 1: EMG recordings showing pseudo-rhythmic spontaneous discharges occurring in bursts in the right extensor digitorum brevis and abductor digiti quinti muscles. terised by pronounced jerks, arising both simultaneously and independently in the different muscles of the lower limb (fig 2). Severe disruption of the sleep pattern was documented on both occasions by the fact that sleep latency was delayed, stage 4 absent, REM sleep was attained only once ( $8 \%$ of total sleep time) and light sleep (stages 1-2) was $67-81 \%$ of total sleep time. The cyclic structure of sleep was lost, and frequent awakenings and arousals were observed.

Case 2 A 74-year-old female had noticed paresthesiae and loss of strength for 4 years first in the left little finger, spreading then to all the other left fingers and, later on, also to the palm and the ulnar aspect of the forearm up to the elbow. She was submitted to surgery twice for this ulnar neuropathy, at the wrist and at the elbow, without any effect. One year later, a sensation of tightness began in

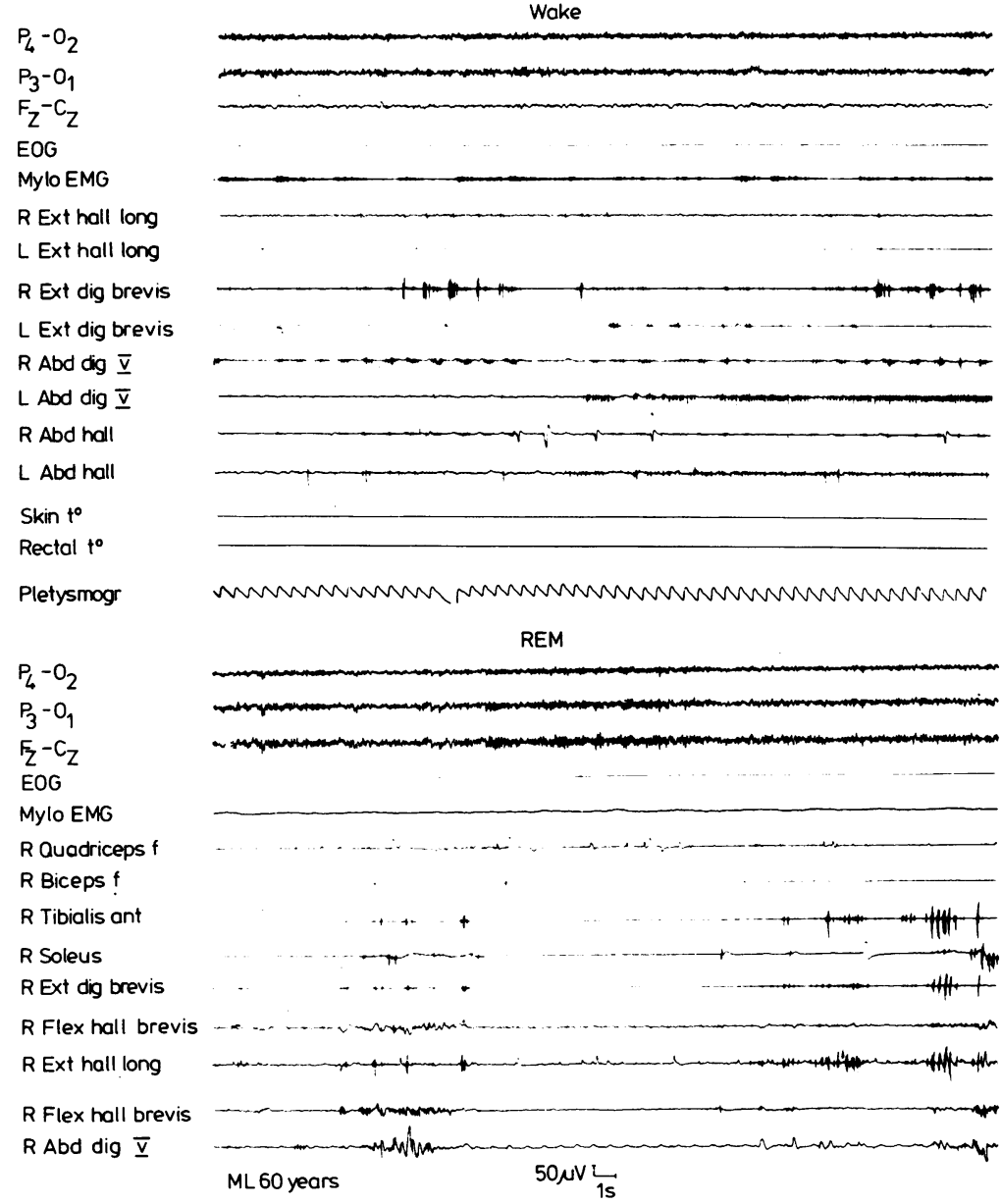

Fig 2 Case 1: during wakefulness (above), irregular spontaneous EMG discharges in the muscles of the lower limbs, sometimes assuming a rhythmic pattern. During REM sleep (below) muscle jerks occur in several muscles of the leg, sometimes in a clonic pattern. 
Wake

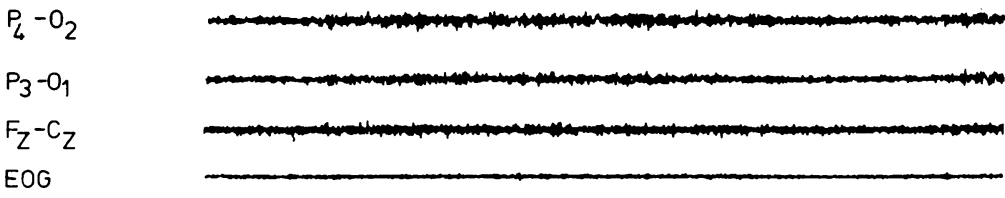

Mylo EMG both feet, associated with tingling paresthesiae, a "rubberlike" sensation under her feet and loss of strength, more on the left. In the month before admission, constant and marked burning pain had also appeared from foot to thigh, especially on the left, and she had also noticed paresthesiae in the fingertips on the right. On examination, there was decreased strength and wasting of both legs distally, associated with loss of sensibility for touch, pinprick, vibration and position sense distally in both legs and in the left ulnar nerve distribution. Tendon jerks were reduced in the upper limbs and absent in the legs. The left plantar response was extensor. Irregular adduction and abduction movements were noticed at rest in the left fifth and fourth fingers. Nearly continuous flexion and extension movements also were observed of all toes and of both feet, occurring irregularly at the ankles and proximal interphalangeal joints. The patient was unable to stop the movements voluntarily for more than a short period of time. WR, VDRL, urinanalysis, full blood count, blood nitrogen and glucose, glucose tolerance test, TSH, $\mathrm{T}_{3}, \mathrm{~T}_{4}$, GOT-GPT, EEG, chest radiography and CSF examination were normal. A Schilling test showed decreased gastrointestinal absorption of both free and intrinsic factor-bound vitamin $B_{12}$. EMG was normal in both lateral vasti but there was chronic partial denervation in the left tibialis anterior. Motor conduction velocity was normal in the peroneal nerve on the left $(45 \mathrm{~m} / \mathrm{s})$ and slightly reduced on the right $(41 \mathrm{~m} / \mathrm{s})$. Sensory conduction velocity was normal $(47 \mathrm{~m} / \mathrm{s})$ but the amplitude of the SAP was severely decreased in the right median nerve $(0.7 \mu \mathrm{V})$.

On a subsequent admission, she had deteriorated. The involuntary movements had now spread to the fingers of both hands and there was continuous burning pain in the legs, radiating along the thighs. Administration of vitamin $\mathrm{B}_{12}, \mathrm{ACTH}$, steroids, benzodiazepines, carbamazepine and antidepressants had no effect. An all-night polygraphic recording showed, during wakefulness, the presence of involuntary jerks, occurring pseudo-rhythmically in the flexor and extensor muscles of the lower limbs and also there were jerks in the muscles of the forearms. The jerks persisted during SWS and also during REM sleep (fig 3). The sleep histogram showed an incomplete cyclic pattern because of the lack of REM sleep, marked latency of the first REM stage with only one episode of REM sleep (5\% of total), a total lack of stage 4 and a prevalence of light sleep stage 1 (47\% of total sleep time) with frequent awakenings.

Case 3 A 76-year-old woman had had diabetes mellitus since age 58 years. At 66 years paresthesiae and sensory loss began in both feet, associated with weakness and fatigue when walking or standing. Diabetic polyneuropathy was diagnosed. Her symptoms slowly worsened, despite insulin therapy, and a trophic ulcer developed at the left ankle. In the last 6 months, severe "cutting", burning and lightning-like pain began, especially during the night, although it was present constantly also during day-time. It affected the right leg especially the toes and ankle up to the knee. Involuntary movements began at the same time in the right foot, consisting of rhythmic jerks at the ankle and more irregular extensionflexion movements of the toes. The movements could not be stopped voluntarily. Lately there had been a worsening of both pain and involuntary movements, despite therapy with ACTH, steroids and analgesics. On examination, 
Awake

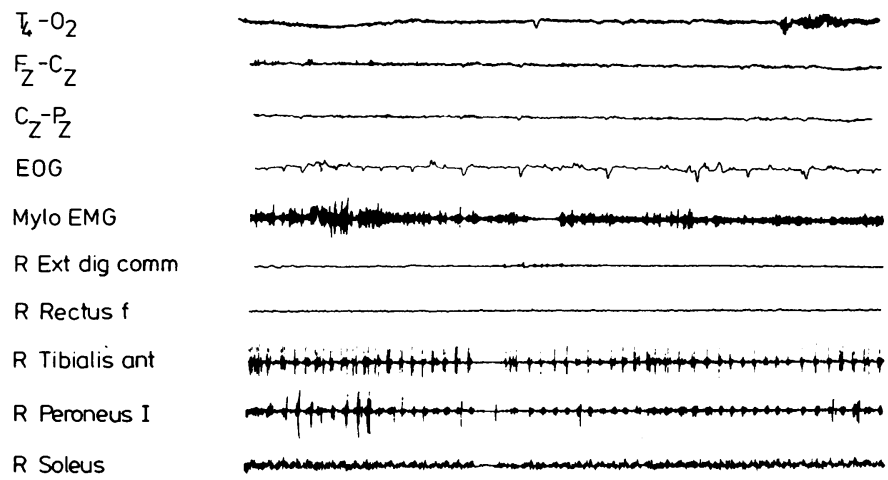

R Ext dig br

L Tibialis ant

L Flex hall

ECG

Plethysmogr
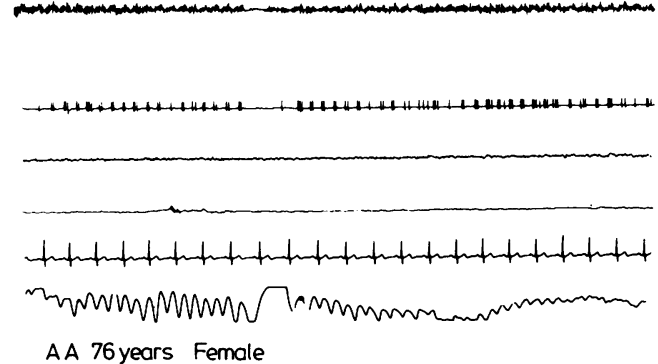

REM
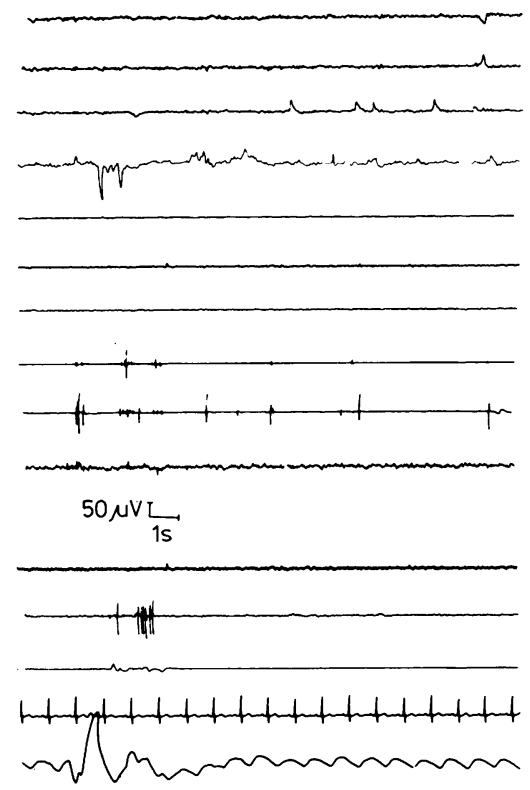

Fig 4 Case 3: during wakefulness (left), periodical jerks in the right tibialis anterior, peroneus longus and extensor digitorum brevis; during REM sleep (right) only sporadic muscle jerks persist.

there was weakness of the distal muscles of both legs, associated with distal hypoaesthesia, more marked for deep sensibility. All tendon jerks were absent. Spontaneous continuous movements were observed in the right lower limb, consisting of large semi-rhythmic eversioninversion at the ankle and sporadic flexion-extension jerks of the toes. Only very sporadic jerks occurred in the left anterior tibial muscle. Both feet were oedematous, and a trophic ulcer was present at the right ankle. Gait was ataxic with a positive Romberg's sign. EMG disclosed denervation and loss of motor units in the left tibialis anterior and abductor pollicis brevis muscles. Distal motor latency was prolonged $(5.2 \mathrm{~m} / \mathrm{s})$ in the left median nerve and the amplitude of the evoked response in abductor pollicis brevis was reduced $(2.5 \mathrm{mV})$. Sensory conduction velocity from the first and third digits to wrist was reduced and the sensory potentials were very small $(28$ and $34 \mathrm{~m} / \mathrm{s} ; 3.9$ and 2.4 $\mu \mathrm{V}$ respectively). The extensor digitorum brevis was totally denervated, and motor conduction velocity in the left peroneal nerve, to the tibialis anterior was reduced (43 $\mathrm{m} / \mathrm{s})$. The sensory potential in the left sural nerve was markedly reduced in amplitude $(0 \cdot 1 \mu \mathrm{V})$ and sensory conduction was slowed $(31 \mathrm{~m} / \mathrm{s})$. An all-night polygraphic recording showed during wakefulness spontaneous activity in the right tibialis anterior and peroneus longus muscles which often was synchronous in the 2 muscles, and rhythmic at about $2 \cdot 5-3 \mathrm{~Hz}$; sometimes it was more irregular, and at other times it was more continuous activity in the right soleus, with irregularly rhythmic bursts in the right extensor digitorum brevis. Only sporadic jerks were seen in the left tibialis anterior. The spontaneous movements were markedly reduced during SWS and also during REM sleep, only isolated sporadic jerks persisting especially in the right peroneus longus and tibialis anterior (fig 4). Sleep histogram showed lack of stage 4 , predominant light-sleep stages $(67 \%)$, total sleep time was decreased and the number of awakenings and arousals increased; REM sleep was normal $(23 \%)$ but rather fragmented.

\section{Discussion}

Troubling pain in the legs, burning in quality and constant was associated with involuntary movements of the toes and feet in these patients. The involuntary movements in the first patient were quite similar to those described by Spillane et al., ${ }^{1}$ ranging from better-coordinated flexion-extension movements to slow quivering or to continuous fanning, clawing and straightening of the toes. In the second case, jerks also occurred in the upper limbs, where they were peculiarly synchronous with those in the feet; they also seemed to involve the ankles more than the toes. In the third case they were almost unilateral, involving, in particular, the ankle in a peculiar pattern of eversion-inversion. Sometimes they had a pronounced rhythmicity, resembling tremor. 
We also found clinical and electrophysiological evidence of peripheral neuropathy in our patients. The first two cases exhibited, on EMG examination, normal or slightly reduced conduction velocities in the presence of severe reduction of the evoked responses, in particular of the sensory action potentials. Such findings usually are ascribed to loss of axons. Slowing in conduction velocity was more marked in the third case and signs of chronic denervation and reinnervation were found on EMG examination of distal muscles. The aetiology of the polyneuropathy was not certain in the first two cases. It may have been due to long-term isoniazid therapy in the first, and to vitamin $\mathrm{B}_{12}$ deficiency in the second. The third case had a diabetic polyneuropathy. Two previous cases described by Spillane et $a l^{1}$ also showed some evidence for peripheral neuropathy, as indicated by segmental demyelination or Wallerian degeneration in a sural nerve biopsy. In one of them, EMG also showed reduced amplitude of the sensory evoked response, and Nathan (personal communication, 1981) has reported occasional slowing of conduction in such cases. "Painful legs and moving toes" should thus be considered a non-specific syndrome, occurring in the setting of several types of peripheral nerve lesions, ranging from root lesions to peripheral nerve trauma and to peripheral neuropathy. ${ }^{2-4}$ The complex, sometimes rhythmic and alternating movements of the leg and foot muscles in our first patient, the jerks synchronous in the upper and lower limbs in the second, the periodic bursts often resembling tremor in the third patient, lend further support to Nathan's hypothesis that the movements are initiated by afferent discharges from the periphery, organised at spinal cord, or even higher levels, with pain arising as a central effect. ${ }^{2}$

By means of polygraphic studies, we showed that the movements are usually reduced but do not disappear during sleep, and that they may continue or even increase during stages of SW or REM sleep.

"Painful legs and moving toes" bears some resemblance to the syndrome of restless legs. However, in the former, pain is severe and causalgic in type, is unrelated to the sleep-wake cycle, is not relieved by leg movements or walking. The spon- taneous movements are different from the jerks typical of nocturnal myoclonus, ${ }^{5}$ and also from the voluntary fidgeting movements that help relieve the distress of restless legs syndrome.

Finally, a striking feature in all our patients was the disruption of the pattern of sleep. The total sleep time was decreased, deep sleep time was scant, there was a total lack of stage 4 , and light sleep stages were preponderant. Brief stages of REM sleep were attained only once in two patients. Spillane et $a l^{1}$ also remarked that some patients with painful legs and moving toes complained of severe insomnia and depression. It is likely that such disruption of sleep is secondary to the distress induced by the pain and/or toe movement.

Addendum After submission of the manuscript, 3 cases of "Painful legs and moving toes", were reported by Wulff, ${ }^{6}$ one of them possibly related to a $\mathrm{L}_{5}$ root lesion. The EMG recording of the spontaneous movements in one case showed alternating 2.5 $\mathrm{Hz}$ bursts in the small flexor and extensor muscles to the second toe, with a maintained reciprocal innervation. Such findings closely resemble ours and further support a "central" origin of the abnormal movements.

\section{References}

' Spillane JD, Nathan PW, Kelly RE, Marsden CD. Painful legs and moving toes. Brain 1971;94:541-56.

${ }^{2}$ Nathan PW. Painful legs and moving toes: evidence on the site of the lesion. J Neurol Neurosurg Psychiat 1978;41:934-9.

${ }^{3}$ Barrett RE, Singh H, Fahn S. The syndrome of painful legs and moving toes (Abstr.). Neurology (NY) 1981;31:4179.

${ }^{4}$ Schott GD. Painful legs and moving toes, the role of trauma.J Neurol Neurosurg Psychiat 1981;44:344-6.

5 Lugaresi E, Coccagna G, Berti Ceroni G, Ambrosetto C. Restless legs, syndrome and nocturnal myoclonus. In: Gastaut H, Lugaresi E, Berti Ceroni G, Coccagna G, eds. The Abnormalities of Sleep in Man. Bologna, A Gaggi Editore 1968;285-94.

- Wulff CH. Painful legs and moving toes. A report of three cases with neurophysiological studies. Acta Neurol Scand 1982;66:283-7. 